\title{
Recent progress on RF orbital angular momentum antennas
}

\author{
Jianchun $\mathrm{Xu}^{\mathrm{a}, \mathrm{b}}$, Yaxian Guo ${ }^{\mathrm{a}}$, Puyu Yang ${ }^{\mathrm{a}}$, Ru Zhang ${ }^{\mathrm{a}, \mathrm{c}}$, Xiaojun Zhai ${ }^{\mathrm{d}}$, \\ Shanguo Huang ${ }^{\mathrm{a}}$ and $\mathrm{Ke} \mathrm{Bi}^{\mathrm{a}, \mathrm{b} *}$
}

${ }^{a}$ State Key Laboratory of Information Photonics and Optical Communications, School of Science, Beijing University of Posts and Telecommunications, Beijing, China;

${ }^{b}$ Beijing University of Posts and Telecommunications Research Institute, Shenzhen, China; ${ }^{c}$ Beijing Key Laboratory of Space-ground Interconnection and Convergence, Beijing University of Posts and Telecommunications, Beijing, China; ${ }^{d}$ School of Computer Science and Electronic Engineering, University of Essex, Colchester, UK Country

*corresponding author: Ke Bi (bike@bupt.edu.cn)

Jianchun $X u$ received the B.Sc. degree in Measurement and Control Technology and Instrumentation Program from the Beijing Institute of Technology, Beijing, China, in 2014. He is currently pursuing his Ph.D. with the Department of School of Science at the Beijing University of Posts and Telecommunications in Beijing, China. His research interests include orbital angular momentum, antenna design, and metasurface.

Yaxian Guo received the B.Sc. degree in physics from Central China Normal University, Wuhan, China, in 2018. She is now pursuing her M.Sc. degree at the Beijing University of Posts and Telecommunications in Beijing, China. Her research interests include orbital angular momentum and metasurface.

Puyu Yang received a B.S degree in communication engineering from Shandong University, Shandong, China, in 2017. He is currently pursuing his Master's degree with the Department of School of Science at the Beijing University of Posts and Telecommunications in Beijing, China. His research interests include data analysis, complex network and computational communications.

$R u$ Zhang received the Ph.D. degree in physics from Sichuan University, Chengdu, in 1997. She is currently a Professor at the Beijing University of Posts and Telecommunications, Beijing.

Xiaojun Zhai received the B.Sc. degree from the North China University of Technology, China, in 2006, and the M.Sc. degree in embedded intelligent systems and the Ph.D. degree from the University of Hertfordshire, U.K., in 2009 and 2013, respectively. He is currently a Lecturer in 
the School of Computer Science and Electronic Engineering at the University of Essex. His research interests mainly include the design and implementation of the digital image and signal processing algorithms, custom computing using FPGAs, embedded systems and hardware/software co-design. He is a BCS member and HEA Fellow.

Shanguo Huang (M'09) received the Ph.D. degree from the Beijing University of Posts and Telecommunications (BUPT), Beijing, China, in 2006. He is currently a Professor with the State Key Laboratory of Information Photonics and Optical Communications, BUPT. He has been actively undertaking several national projects, authored three books and over 150 journals and refereed conferences, and authorized 18 patents. He was a recipient of the Beijing Higher Education Young Elite Teacher, the Beijing Nova Program, the Program for New Century Excellent Talents in University from the Ministry of Education, from 2011 to 2013, and the National Science Fund for Excellent Young Scholars, in 2016. His current research interests include the networks designing, planning, the traffic control, and resource allocations, especially network routing algorithms and performance analysis.

$\mathrm{Ke} B i$ received the Ph.D. degree from the Nanjing University of Aeronautics and Astronautics, in 2012. From 2012 to 2014, he was an Assistant Researcher with Tsinghua University. He is currently an Associate Professor with the Beijing University of Posts and Telecommunications. His research interests include information functional materials and devices, and electromagnetic metamaterials and devices. 


\section{Recent progress on RF orbital angular momentum antennas}

In the last five years or so, there has been a great interest in RF OAM to address the contradiction between the lack of wireless spectrum resources and the continually growing demands of the bandwidth. Recent developments and technological advancements in antenna design, material science, and integrated circuits have enabled miniaturized, multiple modes, tunable in radio frequency (RF) orbital angular momentum (OAM) antenna designs. From conventional methods such as spiral phase plate and reflector antenna to antenna array and metasurface, the working principle and structures are introduced. To improve the performance of RF OAM antennas, several innovative physical concepts, including multiple-input multiple-output, circular polarization rotation, PIN diodes dynamic regulation, and so on have been explored for the designs of RF OAM antenna designs. In this paper, we present an up-to-date survey on the development of RF OAM antenna technologies. We provide details on structure designs and theoretical analyses. The evaluate method and advantage analyses are also included. Open issues and development trends are explored as a source of inspiration towards the future better outcome of RF OAM antenna designs.

Keywords: orbital angular momentum; radio frequency; antenna

\section{Introduction}

In modern communication systems, multiplexing technologies based on amplitude, frequency, and polarization are usually using to increase the transmission effect [1-4]. However, limited by the finite spectrum resource, the capacity, and spectral efficiency cannot be infinitely increased by the multiplexing/demultiplexing technologies $[5,6]$. Nowadays, with the rapid development of microwave technology, the lack of wireless spectrum resources and continually growing demands of the bandwidth are becoming increasingly prominent. Therefore, it is essential and extremely urgent to figure out a feasible method to solve this problem [7-9]. Fortunately, one potential way based on orbital angular momentum (OAM) beam has been proposed after the massive efforts of scientists [10-14]. Importantly, this method can offer a new degree of freedom and has 
the potential to provide infinite channels for wireless communication at radio frequency (RF) $[15,16]$.

In the optical regime, the OAM has been widely applied in many fields, such as imaging, nano-particle manipulation, and optical communicating, owing to its special electromagnetic properties [17-22]. The total angular momentum consists of spin angular momentum (SAM) and OAM. The SAM of light was anticipated by Poynting in 1909 and demonstrated by Beth in 1936 [23,24]. A light beam carrying OAM was recognized by Les Allen in 1992 and described by $\exp (i l \varphi)$, where $l$ is the topological charge, $\varphi$ is the azimuthal angle $[25,26]$. It was shown that each photon carries an OAM of $l \hbar$ ( $\hbar$ is the Planks constant $h$ divided by $2 \pi$ ) [27]. In contrast, a circularly polarized light beam has a SAM of $\pm \hbar$ per photon [28]. The SAM is associated with the circular polarization while the OAM is dependent on the field spatial distribution. The helical phase front is an obvious characteristic of the OAM beams. Owing to the existence of singular-phase null regions, the intensity profiles of the OAM beams exhibit doughnut shapes upon propagation [29]. Compared with the SAM which has only two possible values, the OAM can theoretically provide unlimited states due to the unbounded integer $l$. Additionally, it was verified that the OAM beams have orthogonal property between different states [30-32]. Thus, the OAM beams have the potential to provide infinite communication channels, increasing the capacity and spectral efficiency of modern communication without increasing the bandwidth [33-35]. Although there are some doubts about the capacity of OAM in increasing the capacity and spectral efficiency, the great potential and wide applications still attract extensive research enthusiasm [36-39].

So far, various methods have been proposed to design high-performance antennas for OAM beams generation. These methods have their defects and limitations, 
which greatly restrict the range of practical applications. It is still reminded a long way to achieve large-scale applications in RF OAM communication. Though much academic literature has been published to describe and discuss multifarious RF OAM antenna designs. Unfortunately, most papers only have explored one or some aspects of the RF OAM antenna design without a comprehensive introduction. Therefore, we present a comprehensive survey of RF OAM antennas to provide the readers with the most recent techniques and up-to-date references in the fundamental studies and technical advance in the field of RF OAM antenna design. This survey pays attention to the structure of the antenna, fundamental principles of OAM generation as well as assessing the quality of generated OAM beams.

The remainder of this paper is organized as follows. In the next section, the conventional method includes spiral phase plane and reflector antenna are presented. Some special antennas, such as circular-shaped antennas using high order modes, threedimensional structures, and metallic cavity radiators, are introduced in Section III. The OAM beam generations based on antenna array are discussed in Section IV. Then, the metasurface is investigated for OAM beam generation in Section V. Finally, Section VI concludes this survey.

\section{Conventional methods}

\subsection{Spiral phase plate}

The spiral phase plate (SPP) is the simplest device to generate OAM beams in RF. Initially, the structure of the SPP is shown in Figure 1(a). One surface of the SPP is flat, and another surface is set as a smooth surface to impart an azimuthal phased term for the transmitted beam. In this case, the thickness of the SPP is azimuthally controlled to form various phase delays for OAM generation. The relationship between thickness and 
azimuthal angle can be described by [15],

$$
h(\phi)=\left(\frac{\phi}{2 \pi}\right) l \lambda /(n-1)
$$

Where $h(\phi)$ is the thickness of SPP, $\phi$ represents the azimuthal angle, $\lambda$ is the wavelength, $n$ is the refractive index of the material. By means of controlling the thickness, the proportion of material in the transmission path can be adjusted, the desired phase delay thus is obtained in turn. Due to the comparison between the material and air, there is a term of $(n-1)$ in the formula. It is obvious that the variation range of the azimuthal angle is from 0 to $2 \pi$. Therefore, the maximum thickness difference is [40],

$$
\Delta h=l \lambda /(n-1)
$$

When the material and operating frequency is fixed, the mode $l$ of generated OAM beam can be changed by the thickness of SPP, such as,

$$
l=(n-1) \Delta h / \lambda
$$

The mode $l$ should be a nonzero integer to show a standard helical wavefront. Increasing the thickness of SPP is a simple method to manufacture the mode of generated OAM beams. However, the volume of the SPP enlarges with the rise in thickness, which is not beneficial to the practical applications. As for the phase of the electromagnetic wave, the $2 \pi$ is a period. Thus, the thickness of the SPP can be compressed by one wavelength for generating higher-order mode. In this way, the SPP is split into several angular regions and the whole plate only has a small thickness as depicted in Figure 1(b). 
Although the structure of this kind SPP looks simple and is easily used, the smooth surface processing is a challenge for the machining tools. For convenience, the smooth surface is approximated by a series of discrete steps. A single material can be milled into the step-shaped SPP. The accuracy near the center region exists a certain error because of the size of the milling tool. Splitting the SPP into some sections is a good solution [41]. Each section is separately processed and then resembled together. In doing so, the process accuracy can be ensured and the mode contamination can also be improved.

The SPP is a passive device that can transform the Gaussian beam into an OAM beam. A horn antenna often used to provide excitation and place in front of the SPP. It is worthwhile to note that the Gaussian beam diameter should not be larger than the diameter of SPP to guarantee the quality of the generated OAM beam. Combining the circular waveguide with the SPP has been presented [42]. As shown in Figure 1(c), the SPP is placed inside the horn antenna. Although the requirement of machining is improved, noise immunity can be enhanced. Thus, a more smooth and regular radiation pattern with lower side lobes is obtained, which means generating purer OAM mode.

The above equations illustrate that the refractive index of the material also is an important influence factor for the mode of the generated OAM beam. By means of drilling holes in the material of SPP, the equivalent permittivity is varied to satisfy the phase delay for OAM beam generation [43]. The structure of the proposed SPP is depicted in Figure 1(d). It is mentioned that the reflection loss should be taken into consideration in the SPP design. Compared with no impedance-matched SPP, impedance matching can effectively improve the reflection loss by more than $20 \mathrm{~dB}$. To further decrease the size, a horn lens antenna with a perforated planar dielectric lens is presented [44]. Figure 1(e) shows the configuration of the proposed antenna. Circle 
apertures with different radius are drilled in the dielectric plate to realize the azimuthal variation of the equivalent permittivity. In this design, a conventional horn antenna and the proposed plate are integrated. This method is simple and effective. However, it is

only demonstrated by the simulated results. The real experiment doesn't be carried out.

The SPP is a traditional method for the RF OAM beam generation. Its merits are the simple structure and easy to use. Therefore, it is often used in the researches about the OAM beams application during the previous study. Some solutions have been proposed to solve the problem of difficulty processing. However, it is obvious that one SPP can only generate one OAM beam with one mode. This disadvantage limits the practical applications.

\subsection{Reflector antenna}

The SPP uses the proportion of two kinds of material in the same transmission patch to introduce the desired phase delay. It is absolute that various length of the transmission patch also results in the phase delay, which provides another way to generate RF OAM beam. Based on the SPP design experience, a reflector antenna with a discrete eight-step staircase is designed to reflect the radio beam for OAM generation [45]. As illustrated in Figure 2(a), eight-steps styrofoam blocks are trimmed into a staircase-like structure. Due to the similar principle, the Equation (3) can be used to describe the mode $l$ after modification.

$$
l=\frac{2 \Delta h}{\lambda} \frac{(N+1)}{N}
$$

where $N$ is the number of the staircase. The patch difference is the two times of the height difference because of the electromagnetic wave reflection. To obtain the maximum fainting inside the OAM beam, a correction term about the staircase number 
is added in this equation [46]. The direct result is that each staircase introduces a $-\pi / 4$ phase difference in turn and the total $-2 \pi$ phase difference is achieved. Therefore, the OAM beam with mode of -1 is generated.

In some experimental verification of OAM modal insulation and point-to-point radio link, a modified parabolic antenna, i.e., Cassegrain antenna is applied to generate OAM beam $[47,48]$. This twisted reflector converts the spherical phase front into a focused OAM beam. The helical surface of the modified parabolic antenna is illustrated in Figure 2(b) and can be described, in the cylindrical coordinate system, by the formula [49],

$$
z(r, \phi)=\frac{l(\phi-\pi) \lambda}{4 \pi}+\frac{\pi r^{2}}{4 \pi F-l(\phi-\pi) \lambda}
$$

where $F$ represents the focus of the reflector, and the $\theta$ is substituted by $\phi$ for unified expression. The material of the reflector antenna's body is ceramic that is covered by copper with a thickness of $0.1 \mathrm{~mm}$ to improve the reflection coefficient. Employing this reflector antenna, the orthogonality between different OAM states and the possibility of increasing data transmission capacity in RF OAM communication were verified. The sintering of ceramic material has complex production processes and the special modified parabolic shape also increases the difficulty. Maybe, the three-dimensional printing technology and styrofoam material can make it easier and decrease the weight of the reflector antenna.

For RF OAM communication, a single OAM state cannot exhibit the superiority in increasing the communication capacity. The multiplexing/demultiplexing technologies and generating multiple OAM beams are the key points. To generate multiple OAM beams, a Cassegrain reflector antenna is designed for the simultaneous generation of three OAM modes [50]. The proposed reflector antenna, shown in Figure 
2(c), composes of an OAM mode mux, a $2 \times 2$ open-ended rectangle waveguide matrix, and a Cassegrain dual-reflector antenna. In this design, the multiplexing and generating of OAM beams mainly depends on the OAM mode mux and open-ended rectangle waveguide. The Cassegrain dual-reflector is used to focus the OAM beams for good radiation pattern and high gain. By modifying the matrix and corresponding OAM mode mux, the reflector antenna can easily be optimized to generate higher OAM modes.

Usually, the parabolic reflector antenna (or Cassegrain reflector antenna) is used to focus the generated OAM beam, unavoidably raising the complexity of the antenna. Thus, some studies are carried out to miniaturize the structure of the reflector antenna [51]. The structure, illustrated in Figure 2(d), is slightly tilted away from the plane to approximate the parabolic surface. And then, a microstrip patch antenna is used to feed the laddered SPP. In this way, the structure is easily built in practice and can simultaneously realize two functions of generating OAM beam and focusing. With the help of mechanical tuning devices, it may be able to dynamically change the mode of the generated OAM beam.

The reflector antenna is a classical method to generate RF OAM beam. Generally, it consists of two sections (a reflector and a feeder). The directionality and high gain are its obvious advantage. Nevertheless, the feeder needs to place in front of the reflector, hindering the transmission of the OAM beam. More importantly, the position placed the feeder is the center of the OAM beam where the electromagnetic wave should have the highest quality. Therefore, the reflector antenna is a conventional method in RF OAM beam generation. 


\section{Special structures of OAM antennas}

\subsection{Microstrip patch antenna}

The microstrip patch antenna has widely applied in modern communication systems due to the excellent advantages such as low profile, lightweight, low cost, easy processing, and so on. The traditional methods mentioned above have their inherent drawbacks in processing and complex structures. Therefore, the microstrip patch antenna is taken into consideration to design a compact RF OAM generator. Inspired by the optical OAM beams generation technologies using high order laser modes, a circularly polarized (CP) patch antenna was present to generate OAM beam at microwave frequencies [52]. As depicted in Figure 3(a), an elliptical patch excited by a single coaxial cable is designed to radiate the electromagnetic wave with $\mathrm{CP} \mathrm{TM}_{n m}$ modes. In the case of circular patch feed by two coaxial cables, the relationship between the operating frequency $f$ and the radius $r$ can be described as:

$$
f=\chi_{n m} c /(2 \pi r \sqrt{\varepsilon})
$$

where $\chi_{n m}$ is the $n$th root of $J^{\prime}(\cdot)=0, J^{\prime}(\cdot)$ represents the derivative of Bessel function of the first kind, $c$ is the speed of light, and $\varepsilon$ is the dielectric constant. For CP $\mathrm{TM}_{n m}$, the components of total radiated field in cylindrical coordinate system can be expressed as:

$$
\begin{aligned}
& E_{x}=A e^{-j(n-1) \phi}-B e^{-j(n+1) \phi} \\
& E_{y}=-j\left(A e^{-j(n-1) \phi}-B e^{-j(n+1) \phi}\right)
\end{aligned}
$$

where $A, B$ are the amplitude of the components, and $A$ is always significantly greater than $B$. Thus, only the first term is considered. These two components both have the same azimuthal phase term $\mathrm{e}^{-j(n-1) \phi}$, which demonstrates that the electric field produced by CP $\mathrm{TM}_{n m}$ mode has helical phase front expected by OAM with mode of (n-1). 
Traditionally, two coaxial feed is employed to obtain circular polarization [53]. As shown in Figure 3(b), a $3 \mathrm{~dB}$ quadrature hybrid is applied in the design of the $\mathrm{CP}$ antenna. The two exciting ports can be individually used to generate right-hand and lefthand $\mathrm{CP}$ wave. According to Equation (6), the different $\mathrm{TM}_{n 1}$ modes can be generated by various radii. Besides, the positions of the two coaxial cables are separated by an angular distance of $90^{\circ} /(n-1)$. In fact, a single coaxial feed can also realize the objective. The circular shape and two coaxial cables can be replaced by an elliptical metallic patch and single coaxial cable for compact structure. In these two kinds of designs, the sign of the generated OAM beam is determined by the $\mathrm{CP}$ properties of right-hand and left-hand.

Based on the same principle of the above two designs, a circularly polarized multimode patch antenna was proposed further for the generation of multiple OAM modes [54]. The proposed antenna depicted in Figure 3(c) has one circle patch and two shorted annular ring patches. Each patch was fed by employing two coaxial cables to separately excite $\mathrm{TM}_{11}, \mathrm{TM}_{21}$, and $\mathrm{TM}_{31}$ mode with circular polarization. In this way, the multiple OAM states with mode $l=1,2$, and 3 were simultaneously generated.

In [55], a circular slot antenna, based on a similar concept to the antenna array, was designed for OAM generation. This antenna is connected in a continuous manner, which distinguishes this work from the OAM antenna array design. The top layer of the proposed antenna is a single circular slot and the most important part is the feeding network (Figure 3(d)). By changing the lengths of each consecutive output port, the desired phase difference can be obtained for generating corresponding OAM states.

Generating OAM beams by using the microstrip antenna has some excellent characteristics, such as easy processing, simple structure, low profile, and low cost. The generation of multiple OAM modes is a great innovation and promotes the development 
of RF OAM based communication. However, dynamic regulation and reconfiguration is the trend of RF OAM antenna design. The fixed modes is their significant shortcoming. In the practical applications, fabricating plenty of antennas to generate different OAM states is a tremendous waste.

\section{2. $\quad$ Ring resonator}

The circular loop antenna is one of the most basic antenna with simple structures. It was demonstrated that a circular traveling-wave loop antenna can generate the RF OAM carried beams [56]. Figure 4(a) shows the configuration for the circular traveling-wave loop antenna. Its main body is a metal ring cavity with wide wall $a$ and narrow wall $b$. Through the calculations of vector potential and electric and magnetic fields, it was demonstrated that all the components of fields have azimuth dependency of $\mathrm{e}^{-j l \phi}$. This is the characteristic of the OAM beam generation. The two ports are used to feed same amplitude but $90^{\circ}$ phase difference signal for exciting the traveling wave in the metal cavity. And the angle between two feeding ports need to satisfy,

$$
\gamma=\frac{(2 m+1) \pi}{2|l|}, m=0,1,2, \ldots, l-1
$$

The transmission direction of traveling waves in the ring cavity is determined by the sign of $90^{\circ}$ phase difference. Therefore, the OAM beam with positive mode or negative mode can be generated when the phase difference between two feeding ports is $-90^{\circ}$ or $90^{\circ}$. In order to radiate out the RF OAM beams, a small circular slot is cut on the top wall of the cavity. In this method, the propagation of the radiated OAM beam is in the axial direction, which may bring some problems of phase singularity and divergence. The propagation in the transverse direction and a ring horn outside the resonator can solve these problems. Therefore, the structure with a slot cut on the 
narrow wall, as shown in Figure 4(b), was proposed [57,58]. Moreover, the mode $l$ of the generated RF OAM beam can be controlled by the metal cavity's structural parameter and is approximately expressed as:

$$
C=\frac{2 l}{\sqrt{\left(\frac{2}{\lambda_{0}}\right)^{2}-\left(\frac{1}{a}\right)^{2}}}
$$

where $C$ is the circumference of the ring resonator, $\lambda_{0}$ is the wavelength in vacuum.

Hitherto, many methods have been proposed to generate RF OAM beam, but good multiplexing structure for RF OAM beam generation is still a challenge. Based on the design of the ring traveling-wave antenna, a dual OAM mode antenna was presented [59]. With the help of 3D printing technology and the electroless copper plating surface treatment process, the proposed antenna was fabricated as illustrated in Figure 4(c). A $2 \times 2$ waveguide hybrid copper was used as a feed network to provide a suitable phase difference. More importantly, the multiple OAM modes will be generated when the two input ports of the waveguide hybrid copper are excited simultaneously. Same as some reflector antennas, a ring focus parabolic reflector can effectively concentrate the generated OAM beams. The transmit distance is the short slab of this design. It has the potential to radiate more multiple RF OAM beams by concentrically stacking the ring resonators $[60,61]$. The propagation characteristics and physical size of the antenna also can be improved by using a coaxial dielectric cavity as shown in Figure 4(d). However, it is obvious that the complexity of the antenna will increase.

\subsection{Dielectric resonator antenna}

The dielectric resonator antenna (DRA), which has many outstanding properties in terms of small size, low loss, high radiation efficiency, and the ability to excite multiple 
modes, has attracted extensive attention in wireless communication. In the introduction of the microstrip patch antenna, it has been verified that the OAM beams can be excited by the high order electromagnetic mode. Similarly, there are two methods used to investigate the cylindrical DRA.

The electromagnet whispering gallery modes (WGM) obtains the same azimuthal wave vector with the OAM modes. Thus, it is possible to generate OAM beams by WGM. To stimulate the WGM, a metal feeding line was placed under a highpermittivity cylindrical resonator and is parallel to the tangential direction of the resonator [62]. The configuration of the DRA is shown in Figure 5(a). Based on the equivalence assumption, the cylindrical DRA is equivalent to a circular loop antenna array which consists of $N$ equidistant electric dipoles. By this means, all the components of electric and magnetic fields can be derived and it is easy to demonstrate the OAM generation. The generated OAM mode can be controlled by changing the effective radius of the cylindrical resonator or operating frequency. Thus, different OAM beams with various modes can be stimulated at different frequencies without changing the structure of DRA.

In [63], the theoretical analysis was carried out by directly deriving the electromagnetic wave expressions of the cylindrical DRA. The schematic of the presented antenna is illustrated in Figure 5(b). Regarding the waveguide modes of a cylindrical DRA, there are three kinds of field modes, including $\mathrm{TE}_{n m \delta}, \mathrm{TM}_{n m \delta}$, and $\mathrm{HE}_{n m \delta}$. As mentioned above, the parameter $m$ is usually set as 1 to stimulate OAM modes. According to the expressions, all components of the electromagnetic field obtain an azimuthal phased term $\mathrm{e}^{j l \phi}$ when the mode $\mathrm{TE}_{n 1 \delta}(\mathrm{n} \neq 0)$ or $\mathrm{TM}_{n 1 \delta}(\mathrm{n} \neq 0)$ or $\mathrm{HE}_{n m \delta}$ is stimulated. 
These two designs have a similar structure but use different theories to analyse. However, the sign of the generated OAM beam is not evidently mentioned. By comparing the feeding lines of these two cylindrical DRA, it is easy to find that the sign of the OAM mode can be controlled by changing the position of feed line (left or right). To the best of our knowledge, there is no design about multiple OAM modes generated or dynamically controlled by this DRA, which is significant for RF OAM beams' practical applications.

The special structures of the OAM antennas are the transition period in the development of RF OAM antenna design. Compared with the traditional methods, these designs have achieved grand developments. However, they also have own obvious drawbacks, which greatly restrict their practical applications. Therefore, they are put in a category.

\section{Antenna array}

\subsection{Normal uniform circular array}

Currently, the utilization of uniform circular array (UCA) is the common way to generate RF OAM beams. For a standard UCA, $N$ elements are equidistantly distributed on a circle with a radius of $a$ and around the beam axis. To generate the OAM mode of $l$, each element are fed with uniform amplitude signal and the phase of $n$th element's feed signal need to satisfy,

$$
\phi_{n}=\frac{2 \pi n l}{N}
$$

We assume that the radiation of each element is isotropic and $N$ is sufficiently large. Then, for a detection point in $p(r, \theta, \phi)$ in the spherical coordinate, the array factor of the UCA can be approximately expressed as [64] 


$$
A F \approx N j^{l} \frac{e^{-j k r}}{r} e^{j l \phi} J_{l}(k a \sin \theta)
$$

where $k=2 \pi / \lambda$ is the wavenumber. Therefore, the radiated electric field can be obtained

$$
E \approx E_{s} \times N j^{l} \frac{e^{-j k r}}{r} e^{j l \phi} J_{l}(k a \sin \theta)
$$

where $E_{s}$ is the electric field radiated by a single element. The existence of the azimuthal phased term $\mathrm{e}^{j l \phi}$ illustrates the OAM beam generation. The mechanism of most UCA is based on this theory derivation. The difference is the way to provide the required phase difference for the feed signals.

The feed network is a common method. Different lengths of the feed lines can introduce different phase for the element antennas in the array [65]. As shown in Figure 6(a), a coaxial feeding and a feed network were applied in the design of UCA. Eight element antennas uniformly distributed on a circumference with a radius of $\lambda$. For generating OAM beam with a mode of 1 , the total phase change should be $360^{\circ}$ around the distribution circle. Therefore, each path towards to the element antenna is $\lambda / 8$ longer than adjacent antenna in sequence. In this method, the $45^{\circ}$ phase delay between the adjacent antennas was introduced in the proposed UCA, which satisfies the Equation (10). The same direction arrangement of the element antennas requires $N$ different paths in the feed network. To simplify the design of the feed network, mirror distribution along the vertical axial was applied. Naturally, the feed network also needs mirror distribution in the same way, which can reduce the half work in the design of feed network. Although the patch lengths of the first element and fifth element are the same, there is a $180^{\circ}$ phase difference between these two element antennas according to the mirror distribution. It should be noted that this $180^{\circ}$ phase difference should be 
considered in the whole UCA not a single element antenna. To generate the contrary OAM mode, the proposed UCA with a mirror process along the horizontal axial can easily realize. The principle of this method is simple and doesn't come up with high requirements for the element antennas. However, the feed network is fixed. If we need to generate different OAM mode, the feed network should be redesigned.

In [66], an original phase shifter was used as the core structure of the feed network and separated with the element antennas. Four element antennas laid on the top layer and the feed network was on the bottom layer. This design can decrease the interference between the feed network's signal transmission and element antennas' radiation. Generally, there is a restriction relationship between the generated OAM mode $l$ and the number of element antennas:

$$
|l|<\frac{N}{2}
$$

At some special conditions, this restriction may be broken [67]. Regarding the feed network with an original phase shifter, its structure is illustrated in Figure 6(b). The sharing part of the path can effectively simplify the design of the feed network. Nevertheless, the problem of the fixed feed network has still remained.

To solve this problem, some coaxial cables were adopted to feed the element antennas [68]. By means of properly tailoring the lengths of coaxial cables, a $45^{\circ}$ phase delay was introduced in the feed network because of the number of element antennas and the desired OAM mode. As depicted in Figure 6(c), the feed signal from one port of vector network analyzer (VNA) was equally divided into eight same feed signals by a Wilkinson power divider. Then, the appropriate phase delay was introduced in these feed signals for feeding the UCA by the proposed coaxial cables. The feature of this design is that the phase-shift device is independent. Once the OAM mode needs to alter, 
using other property coaxial cables can accomplish the goal without redesign the whole feed network or UCA.

One interesting method to introduce phase delay was described in [69] by utilizing the vertical interval. As illustrated in Figure 6(d), the element antennas uniformly distributed on a circumference but placed on different vertical planes. The difference in spatial transmission distance also can alter the phase of the radiated electromagnetic wave. There are eight antennas in the UCA. For generating OAM mode of 2 , the decreasing height of each element antenna should be $\lambda / 4$. According to the period of electromagnetic wave, the element antennas placed on the same vertical plane obtain $2 n \pi$ phase difference, $n$ can be an arbitrary integer. Due to the limitation of elements' size, the distribution radius is set as $2 \lambda$. This distribution seems too large according to the discussions about the radius of UCA in [64]. The theoretical analysis of the UCA with vertical interval has expatiated in [70]. More importantly, it was demonstrated that the OAM mode can be controlled by altering the exiting frequency when the array is fixed. The generated OAM mode can be described as following,

$$
l=m, \text { when } f=m f_{0}
$$

Here, $m$ is the integer, $f_{0}$ is the frequency responded to $l=1, f$ represents the exiting frequency. It is not difficult to understand the mechanism. When $f=f_{0}$, the total phase delay $2 \pi$ was obtained and then the OAM mode $l=1$ is generated. The wavelength will decrease to $\lambda_{0} / m$ because of the exiting frequency of $m f_{0}$. The spatial distance is fixed. Therefore, the total phase delay changes to $2 m \pi$. According to Equation (10), the OAM mode $m$ is obtained. This idea was also adopted in [71] to generate dual-band OAM UCA.

In 2016, high-order OAM mode at the X-frequency band was firstly generated [72]. Its schematic configuration is depicted in Figure 7(a). The OAM mode $l$ from 0 to 
7 can be generated by the proposed UCA. In this design, digital attenuators and phase shifters were applied to feed the UCA. The programmable control brings great convenience in practical applications [73]. Any OAM mode satisfied Equation (13) can be generated by the proposed UCA without changing the structure of UCA. Even, increasing the device number can generate higher-order OAM modes.

These above methods are focused on the development of UCA's feeding technology. Compared with other schemes, phase shifter has tremendous superiority. Based on the phase shifter, our group designed a wideband microstrip antenna to improve the performance of UCA in generating OAM modes [74]. In Figure 7(b), an Fshaped patch and some long-strip patches were designed to obtain wideband property. Except for generating different OAM modes, the UCA with wideband property can generate the OAM beams within the scope of the bandwidth.

The doughnut-shaped intensity profiles and vortex phase distribution are the obvious features of the OAM beams. One specific index should be presented to evaluate the quality of generated OAM beams. The purity or azimuth spectrum was put forward $[75,76]$. Generally, the generated OAM beams can be regarded as the compound of multiple OAM modes. According to the principle of Fourier transform, the purity of each mode can be expressed as follow,

$$
p(l)=\frac{1}{2 \pi} \int_{0}^{2 \pi} \psi(\phi) d \phi e^{-j l \phi}
$$

where $\psi$ is the phase distribution, $\phi$ represents the azimuthal angle.

\subsection{Antenna array for multiple OAM modes}

It is important to generate multiple OAM modes for OAM based wireless communication. The Roman lens is a viable approach to feed the UCA owing to the 
multiple-input multiple-output (MIMO) characteristics. Different multiple OAM modes can be achieved by employing various Roman lens and responding element antennas [77,78]. Their geometry architectures are shown in Figure 8(a) and (b). In Figure 8(a), the lens body and the antennas are segregated by the common ground. Another one used the coaxial cables to connect. These designs aim to eliminate the interference for improving the performance of UCA. Traditionally, the Roman lens is used for linear electronically scanned array. When a single input port is fed, a phase difference is generated successively. Therefore, a specific OAM mode will be generated. The quantity of viable modes is the same as the number of input ports. If multi-input ports are simultaneously fed, the multiplexed OAM modes can be generated. As shown in Figure 8 (a), the OAM mode $l= \pm 2, \pm 1,0$ and their multiple modes can be obtained. The OAM mode $l= \pm 1,0$ and their multiple modes can be realized by using the Roman lens shown in Figure 8(b). Besides, the demultiplexing process also can be finished by using the Roman lens.

In [79], two sets of microstrip feed networks were applied to excite two orthogonal polarization. As illustrated in Figure 9(a), an M-shaped feedline is along the $y$-axis and another $\mathrm{U}$-shaped feedline is along the $z$-axis. Four element antennas were used to achieve the OAM beams generation. If port 1 is excited, the phase delay caused by length difference of the path and the mirror structure can be $\left[0^{\circ},-90^{\circ},-180^{\circ},-270^{\circ}\right]$, which indicates the generation of OAM mode $l=-1$. Similarly, the OAM mode of $l=1$ can be generated when port 2 is on work. Since these two-port are stimulated simultaneously, two orthogonal OAM modes with two polarized directions will be generated. It is worthy to note that the way of feed in this design was employing the aperture couple. The apertures and copper plate can enhance the directivity and reflection coefficient of the element antenna. 
The above method adapted two polarization to obtain a good isolation effect. However, it is hard to eliminate interference between two kinds of feedline due to the coplanar structure. Therefore, two sets of feed networks segregated by common ground were presented in [80,81]. As depicted in Figure 9(b), an eight-dividing feed network and sixteen element antennas were placed on the top layer and a reversal feed network was distributed on the bottom layer. To design the appropriate feed network, T-shaped power dividers and U-shaped phase delay microstrips were applied to divide the feed signal and introduce the desired phase difference. The path difference introduces demanded phase difference for generating specific OAM mode and the mirror feed network excites contrary OAM mode. This two-input UCA can generate two OAM modes of \pm 1 . In the design of dual-modes UCA, two feed networks can be segregated by the common ground. However, what about the triple or more OAM modes? To generate more OAM modes, more feed networks and multilayer structures are required. Recently, a four-mode OAM antenna array was designed by employing three feed networks. It is obvious to see the increase in complexity.

A configurable antenna array for generating multiple OAM modes was proposed in [82]. Figure 9(c) shows the prototype of the proposed antenna array. From this picture, four PIN diodes were adapted to control the signal circuit in the feed network. Two working states can be switched by opening or closing the specific PIN diodes, which results in a successive $\pm 90^{\circ}$ phase difference. The value of the phase difference is determined by the path difference. The current direction can be controlled by the PIN diodes. When PIN 1 and 4 are open, PIN 2 and 3 are closed, the state is along clockwise. On the contrary, another state is along counter-clockwise. Due to the symmetric structure, the phase difference is fixed but the states can be altered. Therefore, only two kinds of OAM mode \pm 1 can be achieved by using this structure. 
Certainly, more PIN diodes and complex feed network are needed for generating highorder or more kinds of OAM modes. In these designs, the PIN diodes were connected to the programmable microcontroller for realizing remote control. The single input port is the obvious feature different from other designs. Recently, a mode-reconfigurable OAM UCA based on a similar mechanism was proposed in [83]. With the help of eightelement antennas and microcontroller, six OAM modes of $l= \pm 1, \pm 2, \pm 3$ have been operated.

Two sets of feed networks and two current states can generate two orthogonal polarization states and contrary OAM modes. Inspired by these two methods, A broadband dual-polarized dual-OAM-mode UCA was further proposed [84]. As illustrated in Figure 9(d), two sets of broadband network with four input ports were designed to feed the UCA. Each network can generate \pm 1 OAM modes with one specific polarization. The property of broadband is a highlight of this design that brings more flexibility for their applications. However, the generation of high-order OAM modes needs to be taken into consideration.

\subsection{Circularly polarized $U C A$}

During the primary stage of the research about OAM UCA, the linear polarization antenna is often used as the element antennas of the UCA. Most theory analysis and derivations are based on the situation of linear polarization. The research about circularly polarized UCA reminds a blank space. Until 2017, it was demonstrated that the circularly polarized antenna can also be used in the UCA for OAM mode generation [85]. A circular polarization can be decomposed into two orthogonal linear polarizations. When the phase of the circular polarization satisfies the demands shown in Equation (10). Two OAM modes with horizontal and vertical polarization can be generated. The chirality of the circular polarization is responding to the sign of 
generated OAM mode. As depicted in Figure 10(a), a two-input feed network was designed to excite dual OAM modes of \pm 1 . For circularly polarized antenna, the essence of the phase variation is the electronic field vector rotation around the geometric center. Therefore, the phase delay can be introduced by rotating the element antenna. Herein, four circular patches are used as the element antennas. For rotating the antennas, it only needs to successively rotate the feed points. There are four element antennas and the desired OAM modes are \pm 1 . Thus, the rotation angle should be $90^{\circ}$.

Then, the circularly polarized UCA has a development process similar to the linearly polarized UCA. In [86], some PIN diodes also used to design the circular polarization and reconfigurable OAM UCA. Here, the so-called circular polarization means the polarization of the element antennas. There is another kind of circularly polarized UCA designed to generate circularly polarized OAM beams [87]. Its configuration is illustrated in Figure 10(b). The aperture-couple was presented to eliminate polarization mismatch. By series of derivations about the $\mathrm{E}$ field, the circular polarization of generated OAM beams has been verified. Each OAM mode theoretically obtains right-hand and left-hand circular polarization. The proposal of this theory effectively improves the polarization matching, promoting the performance of OAM in wireless communication.

Compared with the linear polarization, circularly polarized UCAs bring great convenience in introducing the desired phase difference for OAM modes generation. However, the fixed feed network, as mentioned before, also is a big problem. The flexibility of the circularly polarized UCA can't be guaranteed. To solve this problem, mechanically reconfigurable UCAs, as illustrated in Figure 10(c) and (d), have been proposed in $[88,89]$. The feed network shown in Figure 10(c) is a simple power divider. It doesn't affect the phase difference between element antennas. Thus, the operating 
frequency of the generated OAM mode also is tunable. These reconfigurable and tunable characteristics in OAM modes and operating frequency show the high performance of the circularly polarized UCA in generating OAM modes. As shown in Figure 10(d), the element antennas in our design were connected by coaxial cables. The phase delay can be realized by rotating the element antennas in real-time. Furthermore, to improve the axial ratio of the circularly polarized antenna, a V-shaped metasurface structure was applied [90].

The antenna array is a relatively mature method compared with those traditional or special OAM antennas. Some excellent properties such as multiple polarization, multiple modes, broadband, and so on seek to cater for the practical applications of RF OAM beams. Therefore, this way has great potential in RF OAM antenna designs.

\section{Metasurface antenna}

Recently, the metasurface has attracted tremendous attention in the electromagnetic field due to its outstanding performance in coding electromagnetic wavefront $[91,92]$. The metasurface is a kind of artificial structure with periodic or quasi-periodic characteristics, which can realize some special properties that don't exist in natural material. Accurately controlling the phase and intensity of the electromagnetic wave makes it suitable for shaped-beams. Especially in OAM antenna designs, it exhibits enormous advantages of ultrathin, easy to process, standard wavefront, and so on. The most important in RF OAM metasurface antennas is the structure of the unit cell which needs to obtain at least $2 \pi$ phase variation and keep the appropriate amplitude.

\subsection{Single mode}

In 2011, a V-shaped metal bar was firstly used in the metasurface design for generating optical OAM modes [93]. The metasurface depicted in Figure 11(a) consists of eight 
sections. The unit cells in each section have the same structure, introducing the same phase delay. For generating OAM mode of -1, eight sections serviced as constituent antennas need to satisfy the $45^{\circ}$ phase delay between adjacent sections. Based on the same principle, a circular metal patch with metallic column or other shapes were employed as unit cell to design the OAM metasurface antenna in the RF band $[94,95]$.

In this way, the metasurface is used as an antenna array to generate OAM mode by dividing eight sections. In fact, it is a waste for metasurface's capability in accurately tailoring phasefront of the electromagnetic wave. By modifying the phase of each unit cell, the incident wave can be directly translated into OAM beams. If the unit cells are sufficient, arbitrary OAM mode can be generated. For example, the OAM mode of $l=1$ was excited at $60 \mathrm{GHz}$ by a multilayer metasurface shown in Figure 11(b) [96]. Generally, conventional SPP only considers the azimuthal phase term $\mathrm{e}^{i l \phi}$. The phase variation in free space is always ignored. A reflective metasurface with three dipoles was proposed [97]. Since then, various shaped patches were proposed in metasurface for OAM mode generation [98-101]. The key point of these designs is proposing the structures unit cells to satisfy the $2 \pi$ phase variation by altering the structure parameters. Of course, it is better to change the fewer parameters. Besides, the generated OAM beam generally has a big divergence angle which is an enormous problem for longdistance transmission. Thus, our group employed the phase gradient to design OAM metasurface antenna for small divergence angle [102].

In terms of the unit cells' arrangement, there are two main types. As illustrated in Figure 12, it includes a rectangular shape and a circular shape [97,103]. In my opinion, the circular arrangement corresponds to the intensity profile. Therefore, the OAM metasurface antenna designs obtain convenience in phase calculations, arrangement, and unit cells number. In fact, there is also a difference in the method of exciting. Like 
the SPP and the reflector antenna, the way of reflection often causes occlusion in the primary axis area. Yet, the way of transmission doesn't have this problem.

\subsection{Multiple modes or reconfiguration}

The multiple and tunable features in real-time are the inevitable trend in RF OAM metasurface antenna designs [104]. It is easy for a single metasurface to generate multiple OAM modes by using the superposition of the aperture field or other methods [105-109]. As depicted in Figure 13(a), dual-modes with different radiation directions were obtained by a single metasurface [110]. For generating $k$ OAM modes with specific directions $\hat{u}_{k}$, the introduced phase variation of $m n$th unit cell should include three terms and can be described as follow,

$$
\phi_{m n}=-\frac{2 \pi}{\lambda}\left|\vec{r}_{m n}-\vec{r}_{f}\right|+\arg \left\{\sum_{k} \exp \left[j\left(\frac{2 \pi}{\lambda} \vec{r}_{m n} \cdot \vec{u}_{k}+l_{k} \Phi_{k}\right)\right]\right\}
$$

Here, the first term and third term are the phase compensation of free space phase delay and azimuthal term of standard OAM beam. In the single-mode metasurface design, these two terms are enough. To generate multiple OAM modes with specific directions, the phase delay about projection in wave vectors' directions should be taken into consideration.

Generally, the electromagnetic properties of the metasurface are determined by the performances of unit cells [111]. To design broadband metasurface, the unit cells should obtain wideband characteristics [112-115]. For example, dual layers with orthogonal structure and five symmetric parallel dipoles, as illustrated in Figure 13(b), were adapted to stimulate multiple independent operating modes [116]. By cautiously adjusting the structure parameters, the broadband metasurface has been designed for multiple OAM modes generation. 
It has been mentioned before that the OAM modes can be achieved by circularly polarized antenna. In the metasurface, it seems simpler as shown in Figure 13(c). Dual layers with split resonant ring structures were applied to generate orthogonal modes for circular polarization [117]. The rotating of unit cells and concentric circles structures are better for generating multiple OAM modes.

As we know, the conductive PIN diodes have always been effective for realizing tunable OAM antenna design. It is of no exception in metasurface designs. Employing the PIN diodes in suitable places of unit cells, the transmitted electromagnetic wave can be controlled by voltage $[118,119]$. As depicted in Figure 13(d), the OAM mode can achieve dynamic regulation with the help of a programmable control board.

\section{Conclusion}

In this paper, a comprehensive survey has been done on the design of RF OAM antennas. The RF OAM is recognized as a great potential technology to infinitely increase the communication capacity and improve the spectrum utilization efficiency. The development of novel and effective antenna design technologies is essential for building RF OAM based communication systems to solve the problem of the growing demands of bandwidth. In this survey, various RF OAM antenna design technologies, mainly including SPP, reflector antenna, antenna array, and metasurface, are introduced and compared.

Moreover, the tradeoffs between different performance metrics such as antenna size, complexity, feed method, polarization, bandwidth, and mode, require the understanding of the individual design principle and controlling parameters. Compared with other methods, the antenna array and the metasurface obviously have excellent performance in simple structure, bandwidth, and tunable characteristics. In fact, the future development trend of RF OAM antennas will focus on real-time adjustable and 
multiple-modes antenna designs. Although the long-distance transmission of RF OAM beams is not mentioned, it is a great restriction for promoting practical applications and also an enormous challenge for researchers. We hope that this survey will be a valuable platform for understanding the recent progress on RF OAM antenna design technologies and motivate further researchers for designing high-performance antennas.

\section{Disclosure statement}

No potential conflict of interest was reported by the authors.

\section{Funding}

This work was supported by National Natural Science Foundation of China: [grant number 51972033, 61774020, 51788104, 51802023, 61690195, 61675103, 61671085, 61605015, 61575028, 51532004, and 51802021]; the Science and Technology Plan of Shenzhen City: [grant number JCYJ20180306173235924]; Key area research plan of Guangdong: [grant number 2019B010937001].

\section{References}

[1] Gnauck A, Winzer P, Chandrasekhar S, et al. Spectrally efficient long-haul WDM transmission using 224-Gb/s polarization-multiplexed 16-QAM. J. Lightwave Technol. 2011;29(4):373-377.

[2] Sano A, Masuda H, Kobayashi T, et al. Ultra-high capacity WDM transmission using spectrally-efficient PDM 16-QAM modulation and C-and extended Lband wideband optical amplification. J. Lightwave Technol. 2011;29(4):578586.

[3] Ryf R, Randel S, Gnauck A, et al. Mode-division multiplexing over $96 \mathrm{~km}$ of few-mode fiber using coherent $6 \times 6$ MIMO processing. J. Lightwave Technol. 2011;30(4):521-531. 
[4] Lei T, Zhang M, Li Y, et al. Massive individual orbital angular momentum channels for multiplexing enabled by Dammann gratings. Light: Sci. Appl. 2015;4(3):e257.

[5] Marzetta T. Massive MIMO: an introduction. Bell Labs Tech. J. 2015;20:11-22.

[6] Elshaari A, Zadeh I, Fognini A, et al. On-chip single photon filtering and multiplexing in hybrid quantum photonic circuits. Nat. Commun. 2017;8(1):379.

[7] Miao P, Zhang Z, Sun J, et al. Orbital angular momentum microlaser. Science. 2016; 353(6298):464-467.

[8] Zhai X, Amira A, Bensaali F, et al. Zynq soc based acceleration of the lattice boltzmann method. Concurr. Comp. Pract. E. 2019;2019:e5184.

[9] Xu J, Bi K, Zhai X, et al. A dual-band microwave filter design for modern wireless communication systems. IEEE Access. 2019;7:98786-98791.

[10] Wang J, Yang J, Fazal I, et al. Terabit free-space data transmission employing orbital angular momentum multiplexing. Nat. Photonics. 2012;6(7):488-496.

[11] Djelouat H, Zhai X, Al Disi M, et al. System-on-chip solution for patients biometric: A compressive sensing-based approach. IEEE Sen. J. 2018;18(23):9629-9639.

[12] Xie Z, Lei T, Li F, et al. Ultra-broadband on-chip twisted light emitter for optical communications. Light: Sci. Appl. 2018;7(4):18001.

[13] Qu W, Liu H, Wang J, et al. Adjustable round-pulse time delayer for roundrobin differential phase-shift quantum key distribution. Opt. Commun. 2019;448:43-47.

[14] Lin S, Wang H, Wu F, et al. Room-temperature production of silver-nanofiber film for large-area, transparent and flexible surface electromagnetic interference shielding. npj Flexible Electronics. 2019;3(1):6.

[15] Yan Y, Xie G, Lavery M, et al. High-capacity millimetre-wave communications with orbital angular momentum multiplexing. Nat. Commun. 2014;5:4876.

[16] Su H, Shen X, Su G, et al. Efficient generation of microwave plasmonic vortices via a single deep-subwavelength meta-particle. Laser Photonics Rev. 2018;12(9):1800010.

[17] Padgett M, Bowman R. Tweezers with a twist. Nat. photonics. 2011;5(6):343.

[18] Bozinovic N, Yue Y, Ren Y, et al. Terabit-scale orbital angular momentum mode division multiplexing in fibers. Science. 2013;340(6140):1545-1548. 
[19] Maguid E, Yulevich I, Veksler D, et al. Photonic spin-controlled multifunctional shared-aperture antenna array. Science. 2016;352(6290):1202-1206.

[20] Vieira J, Trines R, Alves E, et al. Amplification and generation of ultra-intense twisted laser pulses via stimulated Raman scattering. Nat. Commun. 2016;7:10371.

[21] Wang J. Advances in communications using optical vortices. Photonics Res. 2016; 4(5):B14-B28.

[22] Chu J, Chu D, Smithwick Q, et al. Encoding and multiplexing of 2D images with orbital angular momentum beams and the use for multiview color displays. Research. 2019; 2019:9564593.

[23] Poynting J. The wave motion of a revolving shaft, and a suggestion as to the angular momentum in a beam of circularly polarised light. Proc. R. Soc. Lond. A. $1909 ; 82(557): 560-567$.

[24] Beth R. Mechanical detection and measurement of the angular momentum of light. Phys. Rev. 1936;50(2):115-125.

[25] Allen L, Beijersbergen M, Spreeuw R, et al. Orbital angular momentum of light and the transformation of Laguerre-Gaussian laser modes. Phys. Rev. A. 1992;45(11):8185.

[26] Jing L, Wang Z, Lin X, et al. Spiral field generation in Smith-Purcell radiation by helical metagratings. Research. 2019;2019:3806132.

[27] Li C, Zhao S. Efficient separating orbital angular momentum mode with radial varying phase. Photonics Res. 2017;5(4):267-270.

[28] Vanacore G, Berruto G, Madan I, et al. Ultrafast generation and control of an electron vortex beam via chiral plasmonic near fields. Nat. Mater. 2019;18:573579.

[29] Zhou J, Zhang W, Chen L. Experimental detection of high-order or fractional orbital angular momentum of light based on a robust mode converter. Appl. Phys. Lett. 2016;108(11):111108.

[30] Willner A, Huang H, Yan Y, et al. Optical communications using orbital angular momentum beams. Adv. Opt. Photonics. 2015;7(1):66-106.

[31] Zhang W, Zheng S, Hui X, et al. Mode division multiplexing communication using microwave orbital angular momentum: An experimental study. IEEE T. Wirel. Commun. 2016;16(2):1308-1318. 
[32] Liu C, Pang K, Zhao Z, et al. Single-end adaptive optics compensation for emulated turbulence in a bi-directional 10-Mbit/s per channel free-space quantum communication link using orbital-angular-momentum encoding. Research. 2019;2019:8326701.

[33] Yu S. Potentials and challenges of using orbital angular momentum communications in optical interconnects. Opt. express. 2015;23(3):3075-3087.

[34] Ren Y, Wang Z, Xie G, et al. Free-space optical communications using orbitalangular-momentum multiplexing combined with MIMO-based spatial multiplexing. Opt. lett. 2015;40(18):4210-4213.

[35] Willner A, Ren Y, Xie G, et al. Recent advances in high-capacity free-space optical and radio-frequency communications using orbital angular momentum multiplexing. Phil. Trans. R. Soc. A. 2017;375(2087):20150439.

[36] Chen M, Mazilu M, Arita Y, et al. Creating and probing of a perfect vortex in situ with an optically trapped particle. Opt. Rev. 2015;22(1):162-165.

[37] Yuan T, Wang H, Qin Y, et al. Electromagnetic vortex imaging using uniform concentric circular arrays. IEEE Antenn. Wirel. Pr. 2015;15:1024-1027.

[38] Aspden R, Morris P, He R, et al. Heralded phase-contrast imaging using an orbital angular momentum phase-filter. J. Optics. 2016;18(5):055204.

[39] Zhao M, Gao X, Xie M, et al. Measurement of the rotational Doppler frequency shift of a spinning object using a radio frequency orbital angular momentum beam. Opt. lett. 2016;41(11):2549-2552.

[40] Schemmel P, Maccalli S, Pisano G, et al. Three-dimensional measurements of a millimeter wave orbital angular momentum vortex. Opt. lett. 2014;39(3):626629.

[41] Schemmel P, Pisano G, Maffei B. Modular spiral phase plate design for orbital angular momentum generation at millimetre wavelengths. Opt. Express. 2014;22(12):14712-14726.

[42] Wei W, Mahdjoubi K, Brousseau C, et al. Horn antennas for generating radio waves bearing orbital angular momentum by using spiral phase plate. IET Microw. Antenna. P. 2016;10(13):1420-1427.

[43] Hui X, Zheng S, Hu Y, et al. Ultralow reflectivity spiral phase plate for generation of millimeter-wave OAM beam. IEEE Antenn. Wirel. Pr. 2015;14:966-969. 
[44] Bai X, Liang X, He C, et al. Design of a horn lens antenna for OAM generation. IEEE Antenn. Wirel. Pr. 2015;14:2081-2082.

[45] Tamburini F, Mari E, Thidé B, et al. Experimental verification of photon angular momentum and vorticity with radio techniques. Appl. Phys. Lett. 2011; 99(20):204102.

[46] Mari E, Anzolin G, Tamburini F, et al. Fabrication and testing of $1=2$ optical vortex phase masks for coronography. Opt. Express. 2010;18(3):2339-2344.

[47] Mari E, Spinello F, Oldoni M, et al. Near-field experimental verification of separation of OAM channels. IEEE Antenn. Wirel. Pr. 2014;14:556-558.

[48] Byun W, Lee Y, Kim B, et al. Simple generation of orbital angular momentum modes with azimuthally deformed Cassegrain subreflector. Electron. Lett. 2015;51(19):1480-1482.

[49] Tamburini F, Mari E, Parisi G, et al. Tripling the capacity of a point-to-point radio link by using electromagnetic vortices. Radio Sci. 2015;50(6):501-508.

[50] Byun W, Kim K, Kim B, et al. Multiplexed Cassegrain reflector antenna for simultaneous generation of three orbital angular momentum (OAM) modes. Sci. Rep. 2016;6:27339.

[51] Huang W, Li J, Wang H, et al. Vortex electromagnetic waves generated by using a laddered spiral phase plate and a microstrip antenna. Electromagnetics. 2016;36(2):102-110.

[52] Barbuto M, Trotta F, Bilotti F, et al. Circular polarized patch antenna generating orbital angular momentum. Prog. Electromagn. Res. 2014;148:23-30.

[53] Mao F, Li T, Shao Y, et al. Orbital angular momentum radiation from circular patches. Prog. Electromagn. Res. 2016;61:13-18.

[54] Zhang Z, Xiao S, Li Y, et al. A circularly polarized multimode patch antenna for the generation of multiple orbital angular momentum modes. IEEE Antenn. Wirel. Pr. 2016;16:521-524.

[55] Gui L, Akram M, Liu D, et al. Circular slot antenna systems for OAM waves generation. IEEE Antenn. Wirel. Pr. 2016;16:1443-1446.

[56] Zheng S, Hui X, Jin X, et al. Transmission characteristics of a twisted radio wave based on circular traveling-wave antenna. IEEE T. Antenn. Propag. 2015;63(4):1530-1536.

[57] Zhang Z, Zheng S, Jin X, et al. Generation of plane spiral OAM waves using traveling-wave circular slot antenna. IEEE Antenn. Wirel. Pr. 2016;16:8-11. 
[58] Zhang Z, Zheng S, Zhang W, et al. Experimental demonstration of the capacity gain of plane spiral OAM-based MIMO system. IEEE Microw. Wirel. Co. 2017;27(8):757-759.

[59] Hui X, Zheng S, Chen Y, et al. Multiplexed millimeter wave communication with dual orbital angular momentum (OAM) mode antennas. Sci. Rep. 2015;5:10148.

[60] Zheng S, Zhang W, Zhang Z, et al. Generation and propagation characteristics of electromagnetic vortices in radio frequency. Photonics Res. 2016;4(5):B9B13.

[61] Zhang W, Zheng S, Hui X, et al. Four-OAM-mode antenna with traveling-wave ring-slot structure. IEEE Antenn. Wirel. Pr. 2016;16:194-197.

[62] Pan Y, Zheng S, Zheng J, et al. Generation of orbital angular momentum radio waves based on dielectric resonator antenna. IEEE Antenn. Wirel. Pr. 2016;16:385-388.

[63] Liang J, Zhang S. Orbital angular momentum (OAM) generation by cylinder dielectric resonator antenna for future wireless communications. IEEE Access. 2016;4:9570-9574.

[64] Mohammadi S, Daldorff L, Bergman J, et al. Orbital angular momentum in radio-A system study. IEEE T. Antenn. Propag. 2009;58(2):565-572.

[65] Bai Q, Tennant A, Allen B. Experimental circular phased array for generating OAM radio beams. Electron. lett. 2014;50(20):1414-1415.

[66] Wei W, Mahdjoubi K, Brousseau C, et al. Generation of OAM waves with circular phase shifter and array of patch antennas. Electron. lett. 2015;51(6):442-443.

[67] Zhao M, Gao X, Xie M, et al. Generation of coupled radio frequency orbital angular momentum beam with an optical-controlled circular antenna array. Opt. Commun. 2018;426:126-129.

[68] Spinello F, Mari E, Oldoni M, et al. Experimental near field OAM-based communication with circular patch array. arXiv preprint arXiv: 1507.06889. 2015.

[69] Liu B, Cui Y, Li R. Delay in space: Orbital angular momentum beams transmitting and receiving in radio frequency. Electromagnetics. 2016;36(7):409-421. 
[70] Yuan T, Qin Y, Cheng Y, et al. Generation of OAM radio beams with modified uniform circular array antenna. Electron. Lett. 2016;52(11):896-898.

[71] Xu P, Zhu C, Cui Z, et al. Design and analysis of dual-band antenna array generating dual-mode vortex electromagnetic waves. Microw. Opt. Techn. Let. 2019;61(10):2275-2281.

[72] Kang L, Liu H, Qin Y, et al. Generation of OAM beams using phased array in the microwave band. IEEE T. Antenn. Propag. 2016;64(9):3850-3857.

[73] Gao X, Zhao M, Xie M, et al. 2D optically controlled radio frequency orbital angular momentum beam steering system based on a dual-parallel MachZehnder modulator. Opt. lett. 2019;44(2):255-258.

[74] Xu J, Zhao M, Ru Z, et al. A wideband F-shaped microstrip antenna. IEEE Antenn. Wirel. Pr. 2016;16:829-832.

[75] Gong Y, Wang R, Deng Y, et al. Generation and transmission of OAM-carrying vortex beams using circular antenna array. IEEE T. Antenn. Propag. 2017;65(6):2940-2949.

[76] Yuan T, Cheng Y, Wang H, et al. Mode characteristics of vortical radio wave generated by circular phased array: Theoretical and experimental results. IEEE T. Antenn. Propag. 2017;65(2):688-695.

[77] Bai X, Liang X, Li J, et al. Rotman lens-based circular array for generating fivemode OAM radio beams. Sci. Rep. 2016;6(1):27815.

[78] Chen X, Zheng S, Zhang W, et al. Free-space radio communication employing OAM multiplexing based on Rotman lens. IEEE Microw. Wirel. Co. 2016;26(9):738-740.

[79] Li H, Kang L, Wei F, et al. A low-profile dual-polarized microstrip antenna array for dual-mode OAM applications. IEEE Antenn. Wirel. Pr. 2017;16:30223025.

[80] Guo Z, Yang G. Radial uniform circular antenna array for dual-mode OAM communication. IEEE Antenn. Wirel. Pr. 2017;16:404-407.

[81] Liu D, Gui L, Zhang Z, et al. Multiplexed OAM wave communication with twoOAM-mode antenna systems. IEEE Access. 2018;7:4160-4166.

[82] Liu B, Lin G, Cui Y, et al. An orbital angular momentum (OAM) mode reconfigurable antenna for channel capacity improvement and digital data encoding. Sci. Rep. 2017;7(1):9852. 
[83] Kang L, Li H, Zhou J, et al. A mode-reconfigurable orbital angular momentum antenna with simplified feeding scheme. IEEE T. Antenn. Propag. 2019;67(7):4866-4871.

[84] Liu B, Cui Y, Li R. A broadband dual-polarized dual-OAM-mode antenna array for OAM communication. IEEE Antenn. Wirel. Pr. 2017;16:744-747.

[85] Bai X, Liang X, Sun Y, et al. Experimental array for generating dual circularlypolarized dual-mode OAM radio beams. Sci. Rep. 2017;7:40099.

[86] Liu Q, Chen Z, Liu Y, et al. Circular polarization and mode reconfigurable wideband orbital angular momentum patch array antenna. IEEE T. Antenn. Propag. 2018;66(4):1796-1804.

[87] Guo Z, Yang G, Jin Y. Circularly polarised OAM antenna using an aperturecoupled uniform circular array. IET Microw. Antenna. P. 2018;12(9):15941600.

[88] Li L, Zhou X. Mechanically reconfigurable single-arm spiral antenna array for generation of broadband circularly polarized orbital angular momentum vortex waves. Sci. Rep. 2018;8:5128.

[89] Bi K, Xu J, Yang D, et al. Generation of orbital angular momentum beam with circular polarization ceramic antenna array. IEEE Photonics J. 2019;11(2):7901508.

[90] Xu J, Hao Y, Bi K, et al. Microwave orbital angular momentum beam generation based on circularly polarized metasurface antenna array. Eng. Sci. 2019;6:30-35.

[91] Wu R, Zhang L, Bao L, et al. Digital metasurface with phase code and reflection-transmission amplitude code for flexible full-space electromagnetic manipulations. Adv. Opt. Mater. 2019;7(8):1801429.

[92] Wang Z, Fu X, Zhang Z, et al. Based metasurface: Turning waste-paper into a solution for electromagnetic pollution. J. Clean. Prod. 2019;234:588-596.

[93] Yu N, Genevet P, Kats M, et al. Light propagation with phase discontinuities: generalized laws of reflection and refraction. Science. 2011;334(6054):333-337.

[94] Xu B, Wu C, Wei Z, et al. Generating an orbital-angular-momentum beam with a metasurface of gradient reflective phase. Opt. Mater. Express. 2016;6(12):3940-3945.

[95] Qi X, Zhang Z, Zong X, et al. Generating dual-mode dual-polarization OAM based on transmissive metasurface. Sci. Rep. 2019;9(1):97. 
[96] Chen Y, Zheng S, Li Y, et al. A flat-lensed spiral phase plate based on phaseshifting surface for generation of millimeter-wave OAM beam. IEEE Antenn. Wirel. Pr. 2015;15:1156-1158.

[97] Yu S, Li L, Shi G, et al. Design, fabrication, and measurement of reflective metasurface for orbital angular momentum vortex wave in radio frequency domain. Appl. Phys. Lett. 2016;108(12):121903.

[98] Lei X, Cheng Y. High-efficiency and high-polarization separation reflectarray element for OAM-folded antenna application. IEEE Antenn. Wirel. Pr. 2016;16:1357-1360.

[99] Zhang Y, Lyu Y, Wang H, et al. Transforming surface wave to propagating OAM vortex wave via flat dispersive metasurface in radio frequency. IEEE Antenn. Wirel. Pr. 2017;17(1):172-175.

[100] Byun W, Choi H, Cho Y. Orbital angular momentum (OAM) antennas via mode combining and canceling in near-field. Sci. Rep. 2017;7(1):12805.

[101] Qin F, Wan L, Li L, et al. A transmission metasurface for generating OAM beams. IEEE Antenn. Wirel. Pr. 2018;17(10):1793-1796.

[102] Xu J, Bi K, Zhang R, et al. A small-divergence-angle orbital angular momentum metasurface antenna. Research. 2019;2019:9686213.

[103] Jiang S, Chen C, Zhang H, et al. Achromatic electromagnetic metasurface for generating a vortex wave with orbital angular momentum (OAM). Opt. Express. 2018;26(5):6466-6477.

[104] Fu X, Cui T. Recent progress on metamaterials: From effective medium model to real-time information processing system. Prog. Quant Electron. 2019;67:100223.

[105] Xu H, Hu G, Li Y, et al. Interference-assisted kaleidoscopic meta-plexer for arbitrary spin-wavefront manipulation. Light Sci. Appl. 2019;8:3.

[106] Wang T, Xie R, Zhu S, et al. Dual-band high efficiency terahertz meta-devices based on reflective geometric metasurfaces. IEEE Access. 2019;7:58131-58138.

[107] Ji C, Song J, Huang C, et al. Dual-band vortex beam generation with different OAM modes using single-layer metasurface. Opt. Express. 2019;27(1):34-44.

[108] Madni H, Iqbal S, Liu S, et al. Fully-control of OAM vortex beam and realization of retro and negative reflection at oblique incidence using dual-band 2-bit coding metasurface. arXiv preprint arXiv:1905.07080. 2019. 
[109] Meng X, Wu J, Wu Z, et al. Generation of multiple beams carrying different orbital angular momentum modes based on anisotropic holographic metasurfaces in the radio-frequency domain. Appl. Phys. Lett. 2019;114(9):093504.

[110] Yu S, Li L, Shi G, et al. Generating multiple orbital angular momentum vortex beams using a metasurface in radio frequency domain. Appl. Phys. Lett. 2016;108(24):241901.

[111] Qin F, Gao S, Cheng W, et al. A high-gain transmitarray for generating dualmode OAM beams. IEEE Access. 2018;6:61006-61013.

[112] Akram Z, Li X, Qi Z, et al. Broadband high-order OAM reflective metasurface with high mode purity using subwavelength element and circular aperture. IEEE Access. 2019;7:71963-71971.

[113] Wang L, Liu S, Kong X, et al. Broadband vortex beam generating for multipolarisations based on a single-layer quasi-spiral metasurface. Electron. Lett. 2019;55(22):1168-1170.

[114] Dong X, Sun H, Gu C, et al. Generation of ultra-wideband multi-mode vortex waves based on monolayer reflective metasurface. Prog. Electromagn. Res. 2019; 80:111-120.

[115] Tang S, Li X, Pan W, et al. High-efficiency broadband vortex beam generator based on transmissive metasurface. Opt. Express. 2019;27(4):4281-4291.

[116] Xu H, Liu H, Ling X, et al. Broadband vortex beam generation using multimode Pancharatnam-Berry metasurface. IEEE T. Antenn. Propag. 2017;65(12):73787382.

[117] Chen M, Jiang L, Wei E. Ultrathin complementary metasurface for orbital angular momentum generation at microwave frequencies. IEEE T. Antenn. Propag. 2016;65(1):396-400.

[118] Wang X, Chen Y, Zheng S, et al. Reconfigurable OAM antenna based on subwavelength phase modulation structure. IET Microw. Antenna. P. 2017;12(3):354-359.

[119] Han J, Li L, Yi H, et al. 1-bit digital orbital angular momentum vortex beam generator based on a coding reflective metasurface. Opt. Mater. Express. 2018;8(11):3470-3478. 


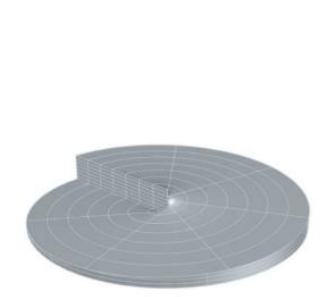

(a)

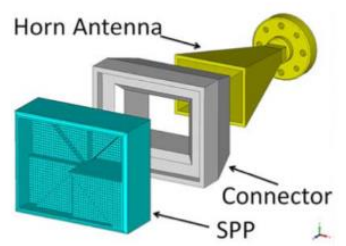

(d)

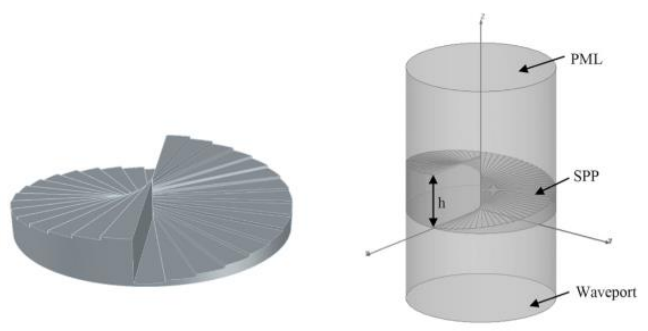

(b)

(c)

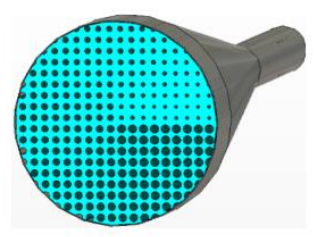

(e)

Figure 1. Schematic of the proposed SPPs with (a) smooth surface [41], (b) several angular regions [41], (c) SPP inside a horn antenna [42], (d) apertures covering the plate [43], and (e) perforated planar dielectric lens [44].

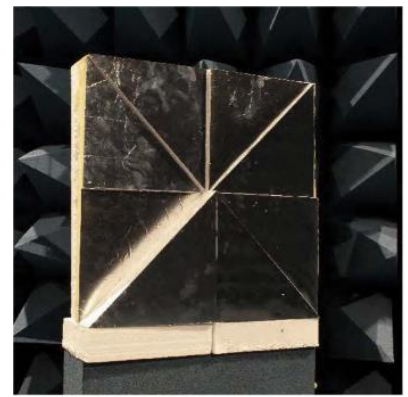

(a)

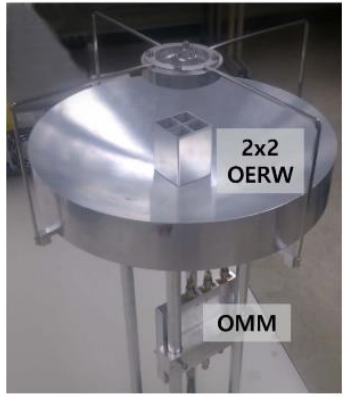

(c)

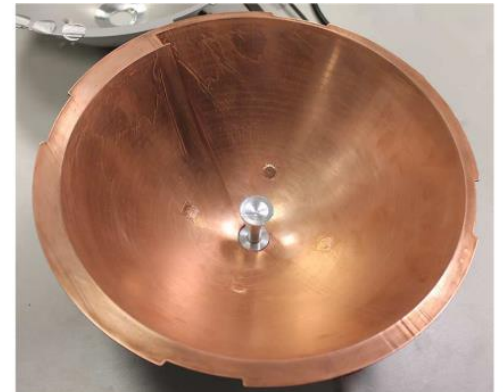

(b)

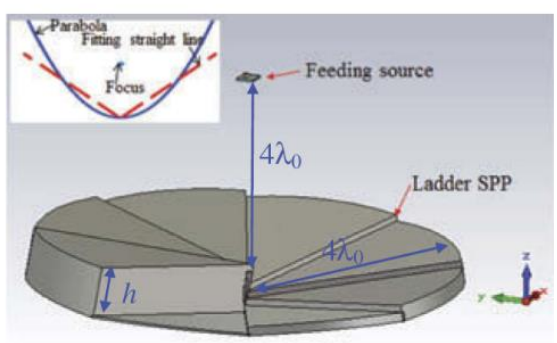

(d)

Figure 2. (a) Reflector antenna with eight-step staircase [45], (b) modified parabolic antenna [47], (c) Cassegrain reflector antenna [50], and (d) laddered SPP [51]. 


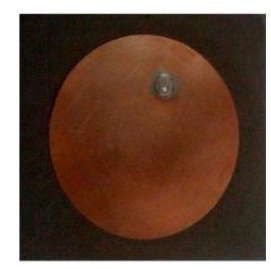

(a)

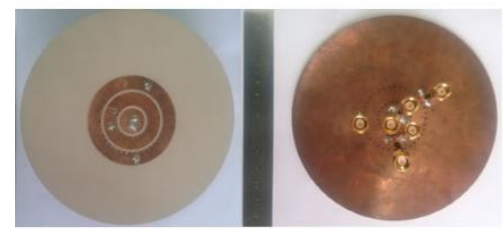

(c)

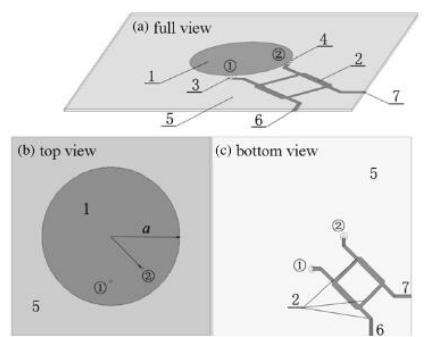

(b)

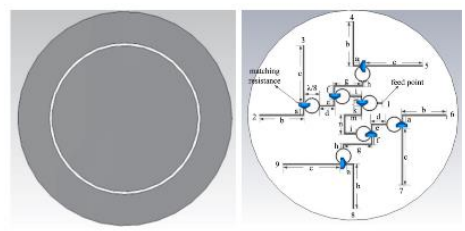

(d)

Figure 3. (a) Microstrip patch antenna with elliptical patch excited by a single feeder [52] (reproduced courtesy of The Electromagnetics Academy), (b) circular patch excited by $3 \mathrm{~dB}$ quadrature hybrid [53] (reproduced courtesy of The Electromagnetics Academy), (c) multiple OAM modes [54], and (d) ring slot antenna with feed network [55].

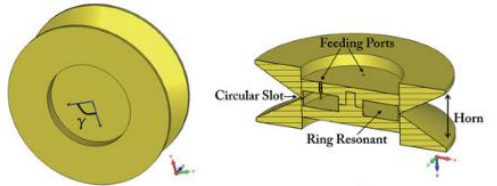

(a)

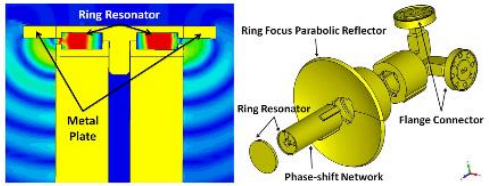

(b)

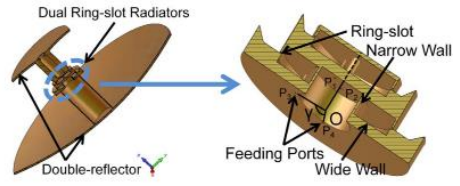

(c)

Figure 4. (a) The slot cut in narrow wall with a horn outside [57], (b) dual OAM modes antenna [59], and (c) multiple OAM modes antenna [60].

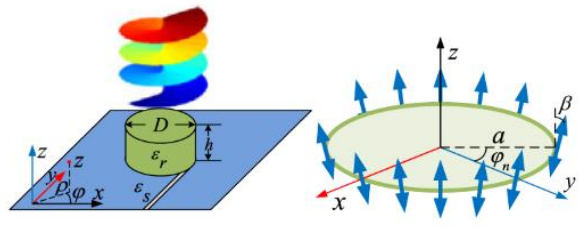

(a)

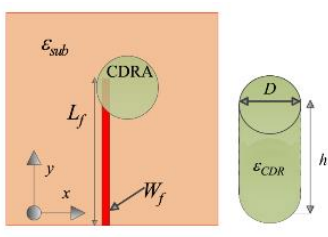

(b) 
Figure 5. (a) High-permittivity cylindrical resonator with whispering gallery modes [62], (b) cylindrical DRA [63].

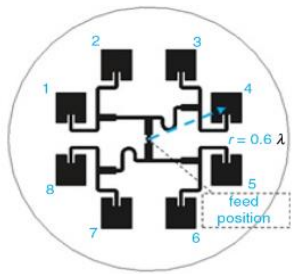

(a)

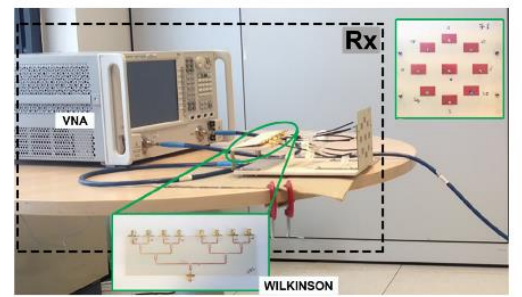

(c)

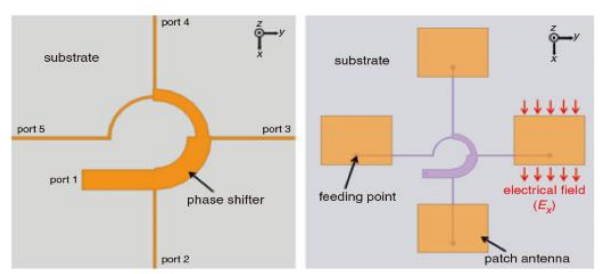

(b)

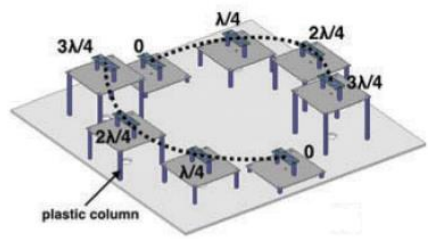

(d)

Figure 6. (a) UCA with mirror feed network [65], (b) original phase shifter [66], (c) tailored coaxial cables [68], and (d) vertical interval [69].

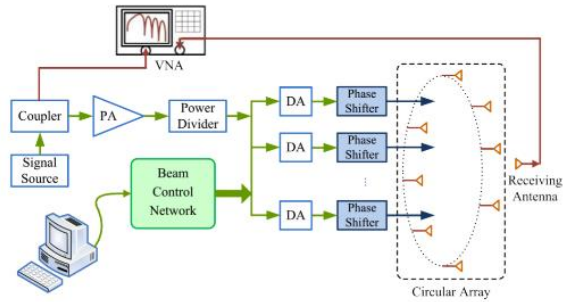

(a)

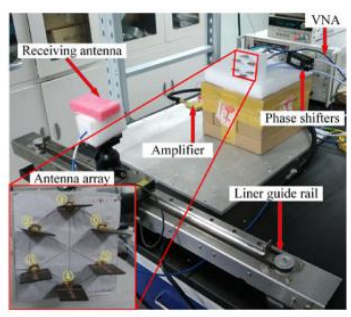

(b)

Figure 7. (a) UCA fed by phase shifters [72], (b) wideband UCA [74].

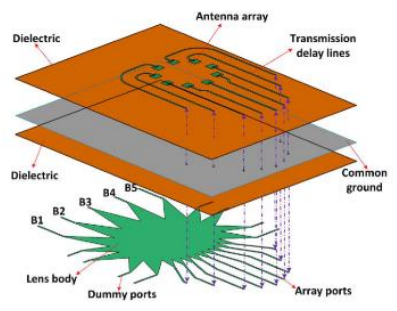

(a)

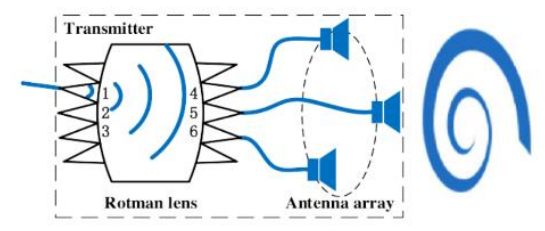

(b) 
Figure 8. (a) UCA with Roman lens segregated by common ground [77], (b) connected by coaxial cables [78].

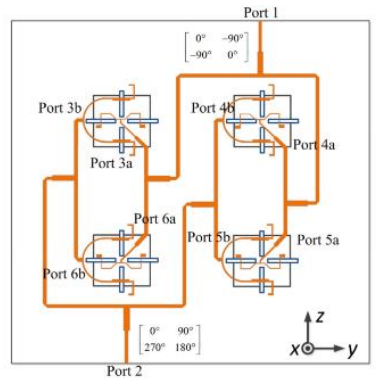

(a)

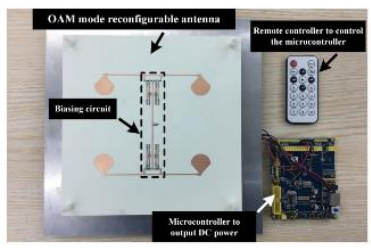

(c)

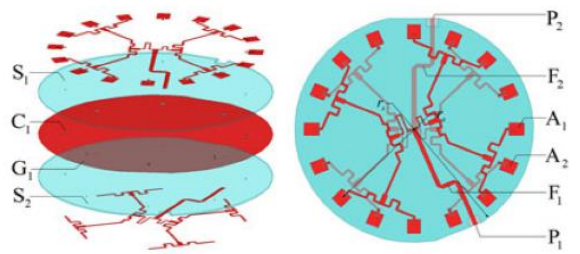

(b)

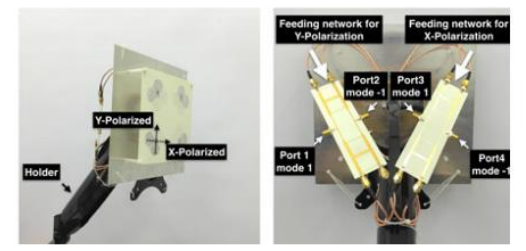

(d)

Figure 9. (a) Dual-polarized OAM modes UCA [79], (b) dual-mode OAM UCA [80], (C) dual-mode OAM UCA with single input [82], (d) dual-polarized dual-mode OAM UCA [84].

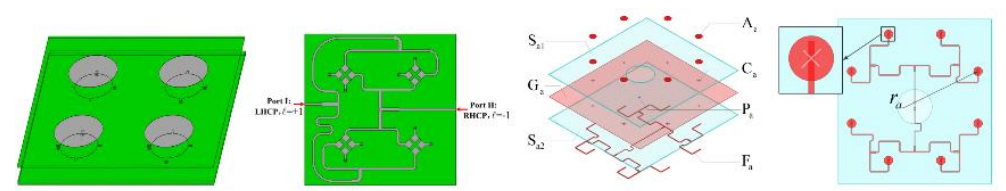

(a)

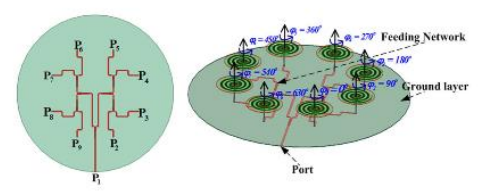

(c) (b)
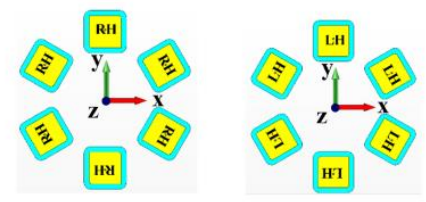

(d)

Figure 10. (a) Circularly polarized UCA with two-input [85], (b) aperture coupling [87], (c) mechanical rotation [88], (d) coaxial cables [89]. 


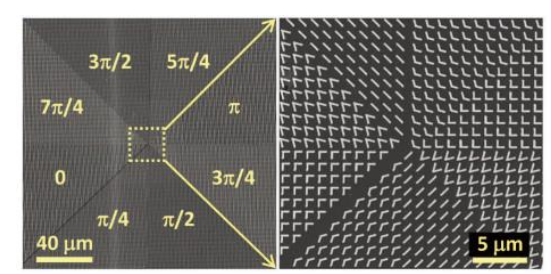

(a)

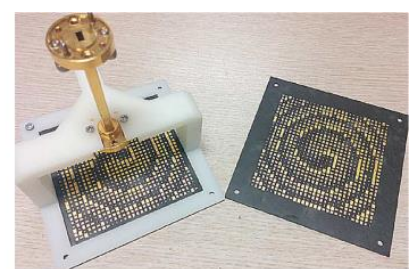

(b)

Figure 11. (a) Metasurface antenna with eight sections [93], (b) direct-translation metasurface [96].

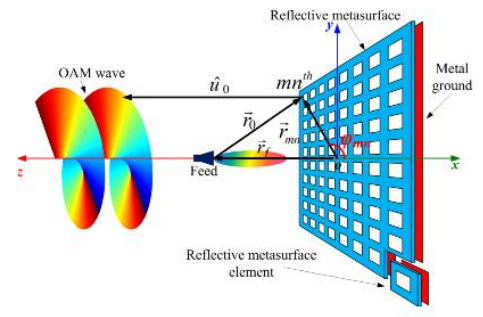

(a)

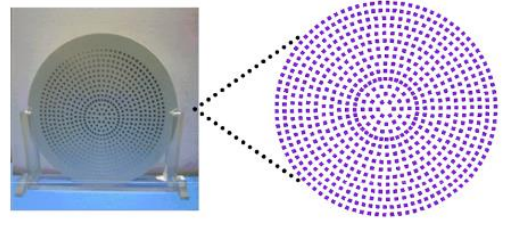

(b)

Figure 12. (a) Metasurface with rectangular arrangement [97] and (b) circular arrangement [103].

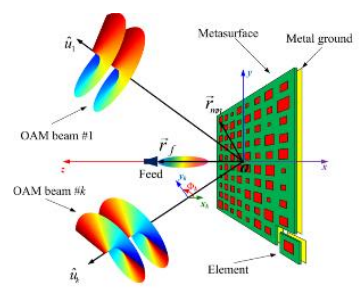

(a)

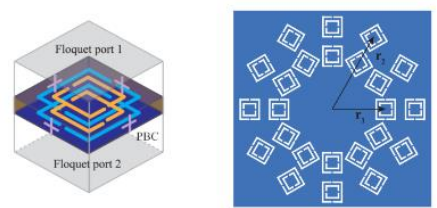

(c)

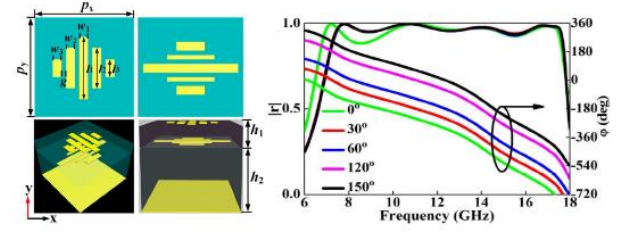

(b)

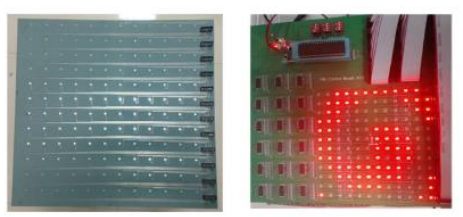

(d)

Figure 13. (a) Multiple modes with different directions [110], (b) broadband unit cell [116], (c) circularly polarized metasurface [117], (d) metasurface with PIN diodes [119] 